\title{
A Rare Case of a Massive Food Bolus Mimicking Lung Cancer
}

\author{
Abdul Karim Arida ${ }^{1}$, Omar Khaddam ${ }^{1}$, Sarah Al Naher ${ }^{1}$, Ashraf Elghul ${ }^{1}$ \\ 1. Internal Medicine, Sheikh Shakhbout Medical City, Abu Dhabi, ARE
}

Corresponding author: Omar Khaddam, omarkhaddam@me.com

\begin{abstract}
Tracheobronchial aspiration is a very common and serious medical condition. It can present acutely with an aspiration pneumonia, or it can be chronic and occur over a long period of time. In some instances, the diagnosis can be missed, and patients may be treated for years for other medical conditions such as asthma, with no significant improvement. We present here a very interesting case of a 69 -year-old gentleman with multiple comorbidities who presented with a fever and shortness of breath. He was initially diagnosed with aspiration pneumonia, but when he did not improve, a bronchoscopy was performed, which showed a mass in the right bronchus suspicious for a carcinoid tumor. However, a biopsy was taken and sent to pathology for analysis, which showed food material. He underwent a rigid bronchoscopy for mass removal, which indeed confirmed that the whole mass was composed of food material as a result of tracheobronchial aspiration.
\end{abstract}

Categories: Internal Medicine, Radiology, Pulmonology

Keywords: aspiration, bronchial carcinoid, food bolus

\section{Introduction}

A rare occurrence but a serious medical condition, tracheobronchial aspiration is when solids or liquids become retained in the airways of the lungs [1]. The clinical presentation of foreign body aspiration can range from chronic insidious lung damage to acute asphyxiation [2]. Common symptoms of aspiration include chronic cough, shortness of breath, and hemoptysis. A history of choking or regurgitation after feeding is a strong pointer towards aspiration; however, not all patients may have these clinical features $[3,4]$. As in our case presentation, there is often no clear history of aspiration, and diagnosis may become a challenge [5]. Several conditions and risk factors increase a patient's risk for aspiration, and these include neurological dysfunction such as seizures, stroke, and Parkinson's disease, alcoholism, facial trauma, and anatomical abnormalities of the pharynx and esophagus [6]. Often, patients with chronic aspiration are misdiagnosed with other conditions such as obstructive airway disease and do not respond satisfactorily to therapy [7]. In this report, we describe the case of a patient with a foreign body in the right bronchus misdiagnosed initially as pneumonia and later as lung malignancy.

Received 08/24/2020

Review began 09/09/2020 Review ended 10/14/2020 Published 10/19/2020

\section{() Copyright 2020}

Arida et al. This is an open access article distributed under the terms of the Creative Commons Attribution License CC-BY 4.0., which permits unrestricted use, distribution, and reproduction in any medium, provided the original author and source are credited.

\section{Case Presentation}

A 70-year-old gentleman who came for a visit from Sri Lanka to UAE in January 2020 presented in the emergency room on February 22, 2020, with complaints of subjective fever and cough productive of whitish sputum for three days prior to presentation. His symptoms were associated with generalized weakness, fatigue, and insomnia. He reported a history of an unintentional loss of $3 \mathrm{~kg}$ of weight secondary to decreased appetite over the past month. There were no symptoms of nausea, vomiting, shortness of breath, dysuria, or abdominal pain. There was no report of difficulty swallowing or coughing or regurgitation with food.

He was a poorly controlled type 2 diabetic with chronic kidney disease stage IIIA3 secondary to diabetic nephropathy and benign prostatic hyperplasia. His last hospitalization was about three months earlier, where he was admitted for investigation of right upper quadrant pain and was found to have gallstone disease. His surgical history is inclusive of a transurethral resection of the prostate about a year earlier, which failed, and he has been on indwelling urinary catheter ever since. His medications are atorvastatin, aspirin, tamsulosin, and gliclazide. He has no known allergies. He has a history of smoking five cigarettes a day for 40 years, but he quit smoking three years ago. The patient denied alcohol consumption or illicit drug use.

On physical examination, he seemed to be uncomfortable and tachypneic with a respiratory rate of 19 breaths per minute. His temperature was 38.2 degrees Celsius, pulse was 104 beats per minute, blood pressure was $152 / 65 \mathrm{mmHg}$, and oxygen saturation was $98 \%$ on $2 \mathrm{~L}$ of oxygen through nasal cannula. He was alert and oriented to time, place, and person, though he seemed to be in mild respiratory distress. He had no signs of anemia or jaundice. Neck examination showed no evidence of jugular venous distention. Chest examination revealed normal heart sounds without any murmurs. Auscultation showed reduced breath 


\section{Cureus}

sounds over the right lower zone with minimal crepitations and dullness to percussion. The abdomen was soft and non-tender without any distention or organomegaly. Neurological examination elicited no focal defects.

His labs showed lymphocytosis with a neutrophilic shift and elevation in inflammatory markers including Creactive protein of $350 \mathrm{mg} / \mathrm{L}$ and procalcitonin of $27 \mathrm{ng} / \mathrm{mL}$. His chest X-ray (Figure 1) showed opacification of the right lower lobe with elevation of the hemidiaphragm. Urine analysis was positive for leukocyte esterase and nitrites. He was admitted for the evaluation and management of urosepsis and healthcareassociated pneumonia, and he was started on piperacillin-tazobactam 2,000 mg IV every 6 hours and azithromycin $500 \mathrm{mg}$ IV every 6 hours. His urine culture came back three days later growing pan-resistant Pseudomonas aeruginosa sensitive only to Colistin; however, that was not started due to his acute renal impairment. Furthermore, his urine was not the likely source of infection since he had been on an indwelling urinary catheter for a long period of time and is therefore expected to be colonized with multiple organisms.

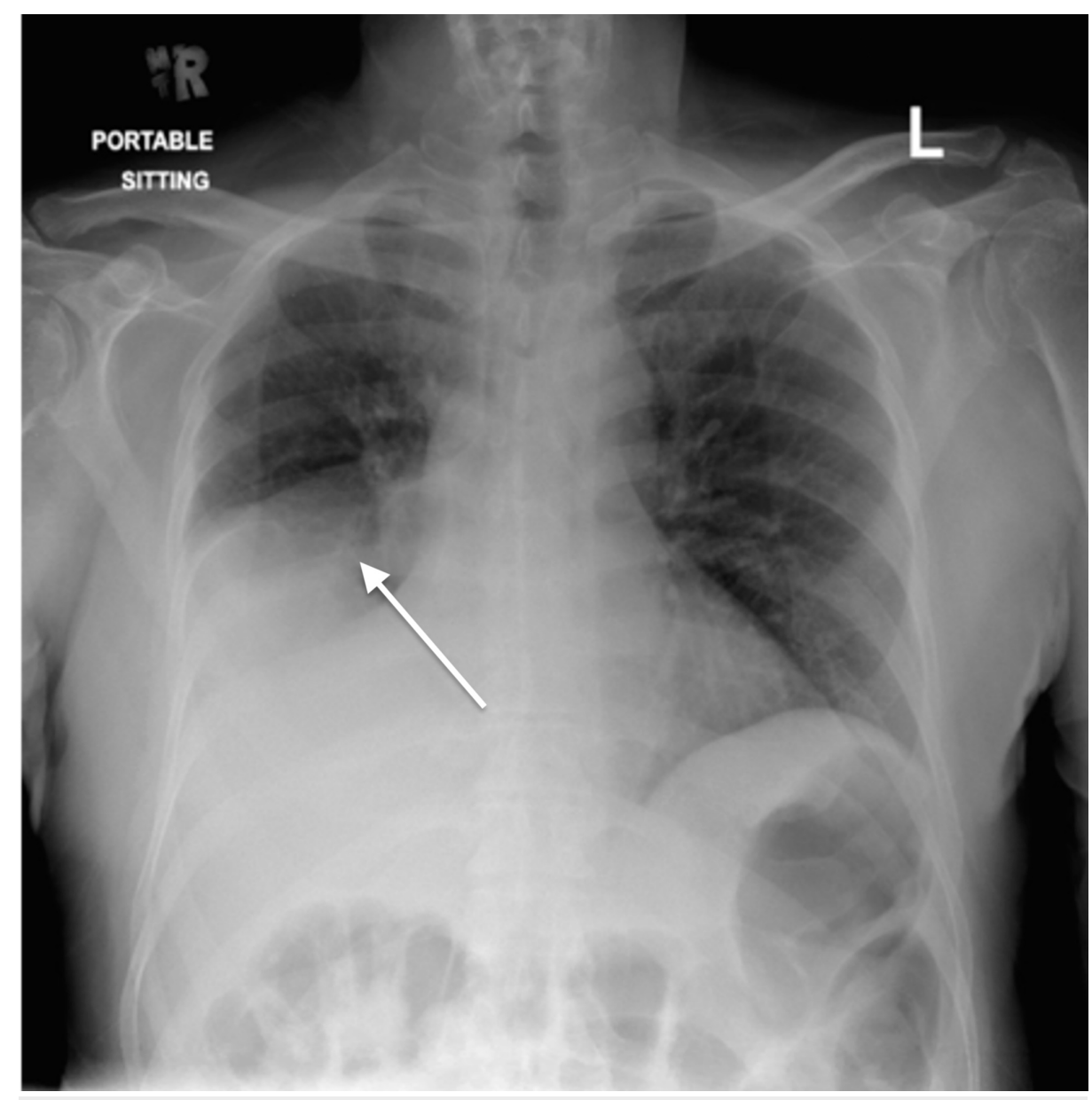

FIGURE 1: Chest X-ray on presentation

Despite being on appropriate treatment for his presumed diagnosis of pneumonia, his respiratory status did not seem to be improving with persistent fever and rising inflammatory markers; his sputum and blood cultures revealed no growth. It was therefore decided to pursue CT of the chest with intravenous contrast for further evaluation of possible malignancy. This was not an easy decision considering his renal impairment, and it was discussed in detail with the patient and his family.

CT of the chest (Figure 2) revealed a $1.4 \mathrm{~cm} \times 1 \mathrm{~cm}$ homogenously enhancing mass in the right bronchus intermedius causing collapse of the right lower lobe in addition to a small right-sided pleural effusion. There was no report of lymphadenopathy or other focal nodules. The findings were suspicious for bronchial carcinoid. On further questioning, the patient reported no symptoms of flushing, diaphoresis, tremors, or diarrhea. Later on, urine 5-HIAA (5-hydroxyindoleacetic acid) test was performed, which came back normal. 


\section{Cureus}

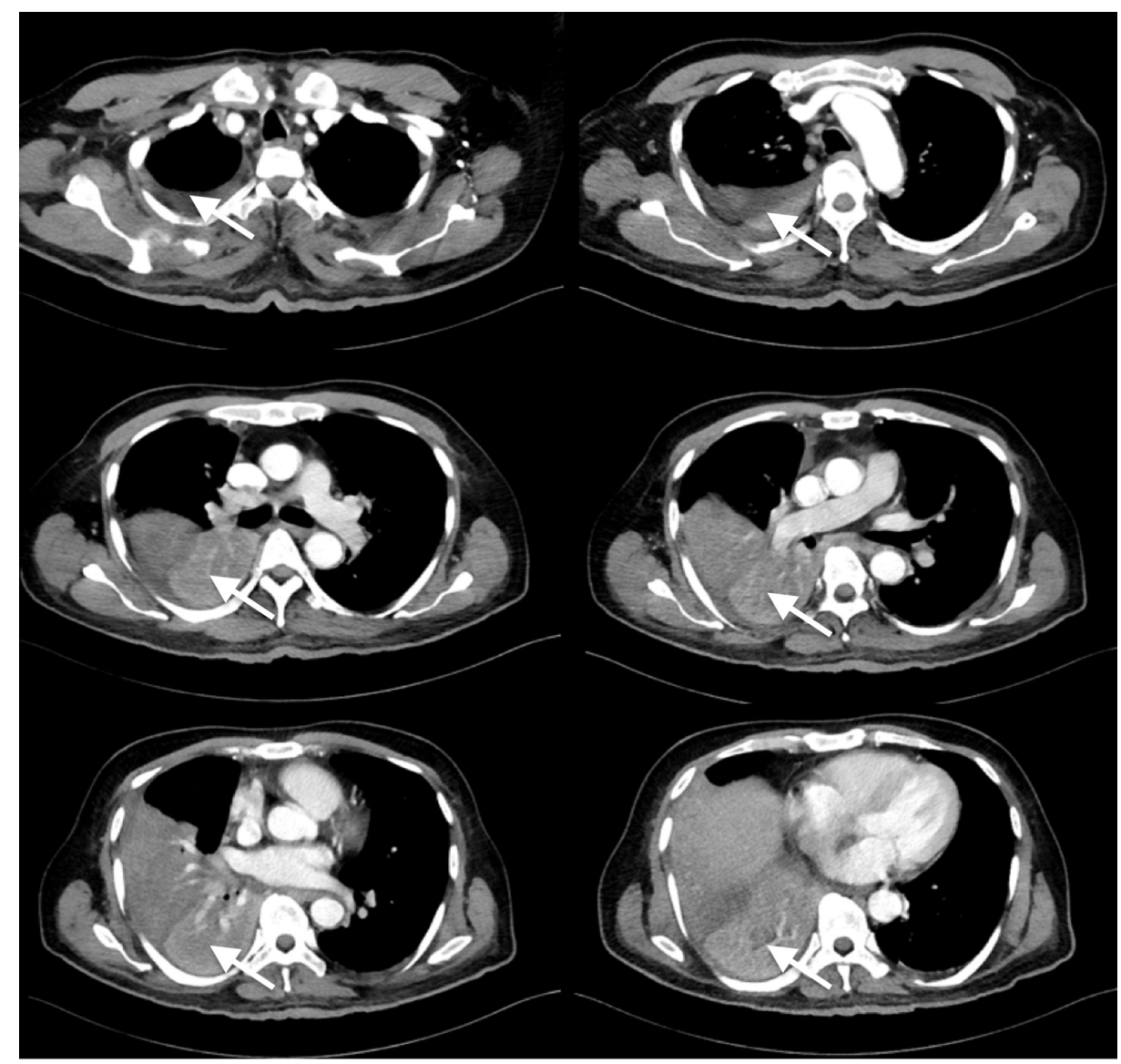

FIGURE 2: CT of the chest showing homogenously enhancing mass in the right bronchus intermedius causing collapse of the right lower lobe in addition to a small right-sided pleural effusion

After discussion with the pulmonologist, the patient was sent for evaluation with bronchoscopy and biopsy of the lung mass. The procedure confirmed the presence of a mass blocking the bronchus intermedius (Figure 3). The biopsy was reported two days later as impacted food material. His antibiotics were discontinued at this point, and he was referred to swallowing and vocal cord evaluation, which showed intermittent disorganized lingual movements and a high aspiration risk. It was recommended that he starts a dysphagia diet with thickened fluids and pureed solid materials. 


\section{Cureus}

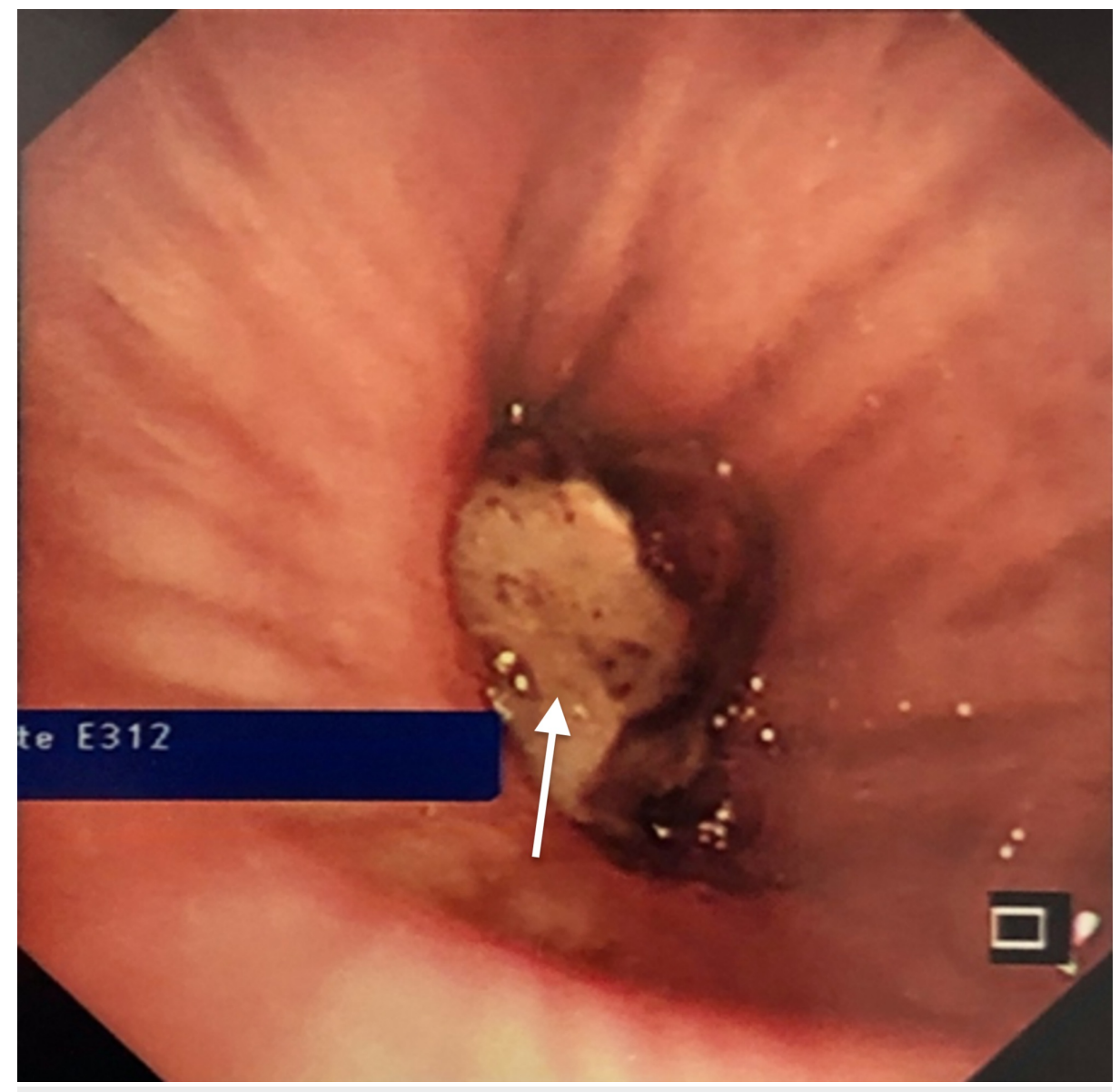

FIGURE 3: Mass seen in the right bronchus during bronchoscopy

The patient later underwent rigid bronchoscopy for the retrieval of foreign material by thoracic surgery. After removal, the patient's white cell count and inflammatory markers started trending down, and he started feeling much better. During his follow-up two months later, a repeat chest X-ray was performed, which showed improvement and re-expansion of the collapsed lung (Figure 4). 


\section{Cureus}

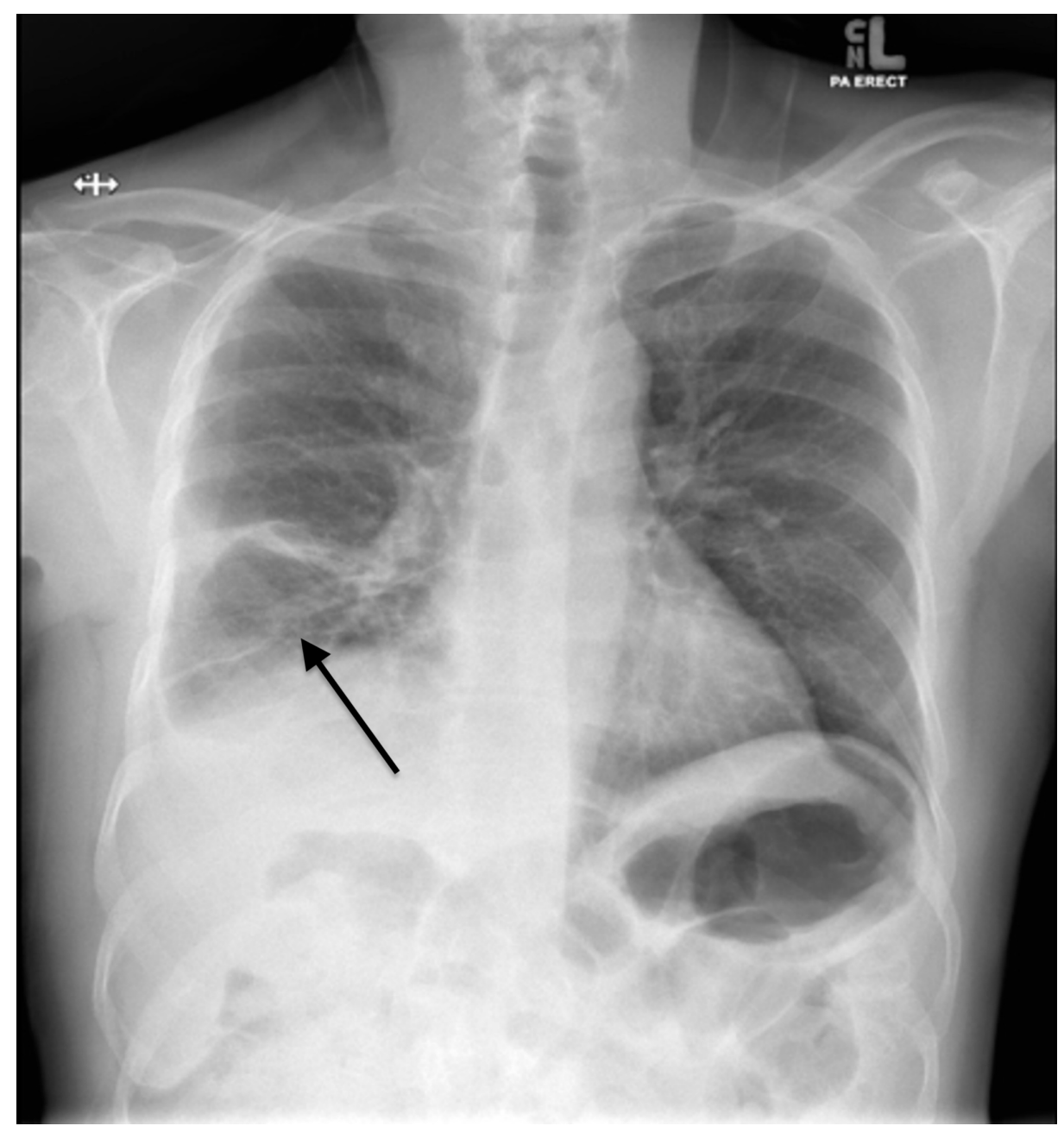

FIGURE 4: Chest X-ray on follow-up after foreign body removal

\section{Discussion}

In healthy adults, foreign body aspiration is uncommon, whereas it more commonly occurs in children and the elderly [3]. Adults who develop foreign body aspiration often have one of several conditions and risk factors, which increase a patient's risk for aspiration, and these include neurological dysfunction such as seizures, stroke, and Parkinson's disease, alcoholism, facial trauma, and anatomical abnormalities of the pharynx and esophagus [6]. The swallowing and cough reflexes are essential defense mechanisms, which protect from aspiration; however, if they get bypassed or suppressed, patients develop an increased aspiration risk $[3,4,7]$. Aspirated foreign bodies may remain in the main bronchi or their branches, leading to obstruction, or they may even reach the lung. Due to its more vertical path, the right main bronchus is the most common culprit [1].

The clinical presentation of foreign body aspiration can vary widely from an indolent chronic cough in up to two-thirds of patients to more life-threatening emergencies including upper airway obstruction with respiratory failure requiring urgent intervention [1]. Patients may also present with intermittent choking sensation associated with a dry cough, which indeed raises the suspicion for aspiration; however, the majority of patients do not present like this [3]. Other clinical features include wheezing, hemoptysis, and shortness of breath [8]. Clinical outcomes can vary from immediate resolution to recurrent pulmonary disease or even death. Sometimes, as is the case in our patient, there are no features from history or clinical assessment to suggest aspiration, and further workup is required [5].

The most commonly aspirated foreign bodies include food particles, broken fragments of teeth, plastic objects, metallic objects including pins, screws, and needles [9]. A series of 3,217 cases over 26 years showed that in children, the most commonly aspirated objects were peanuts, vegetable matter, and toy parts. However, the series demonstrated that adults more commonly aspirated food particles, medication tablets, and dental pieces [10].

Foreign bodies that are radiologically opaque can be directly visualized on chest X-ray unless obscured by 
parenchymal changes [11]. Other radiological features of foreign body aspiration include atelectasis, unilateral hyperinflation, unresolving pneumonia, and localized bronchiectasis [11]. In addition, CT scans of the chest may reveal an intra-parenchymal or intra-bronchial mass, as was the case in our patient [8].

If the diagnosis is not clear after initial workup, the definitive diagnosis of foreign body aspiration can be confirmed when visualized directly using indirect laryngoscopy or bronchoscopy [12]. Furthermore, the foreign body can be retrieved using grasping forceps during fiberoptic or rigid bronchoscopy, as was the case in our patient $[3,6]$. However, in cases of chronic aspiration, the foreign body may not be visualized clearly on bronchoscopy, which would instead show features of tissue reaction to the foreign body including endobronchial stenosis, granulation tissue, strictures, and edema [1,12].

As in our patient, establishing the diagnosis of foreign body aspiration can pose a challenge due to multiple reasons [13]. A large proportion of patients may not give a clear history of aspiration or of any risk factors that would suggest aspiration. Furthermore, some patients may present months to years after the aspiration event [14]. Moreover, some patients may develop subtle clinical symptoms and their aspiration may remain undetected for many years [15].

In some patients, aspiration is not recognized and they are misdiagnosed as asthma, emphysema, chronic pneumonia, or malignancy, similar to what happened with our patient $[8,16]$. This should raise our suspicion for occult foreign body aspiration in cases of chronic or recurrent pneumonia, which do not respond to antibiotic therapy [16]. In other instances, foreign bodies may be incidentally discovered when bronchoscopy is performed for evaluation of a chronic cough, hemoptysis, or an unresolving pneumonia [17].

Removal of the foreign body is usually required for definitive treatment, and the first step in doing so is a flexible bronchoscopy [18]. However, this is successful initially in around $90 \%$ of patients, who will require further management with a rigid bronchoscopy, as was the case in our patient. Rigid bronchoscopy is usually pursued in cases where flexible bronchoscopy fails or is found inadequate for simultaneous safe extraction and airway management [18]. If bronchoscopy shows significant granulation tissue or airway stenosis and extraction is not possible, other modalities such as endobronchial ablation or cryotherapy may be attempted [19].

Delaying the diagnosis of foreign body aspiration, which results in inappropriate management, can lead to chronic complications including recurrent pneumonia, bronchiectasis, strictures, and development of inflammatory polyps at the site of obstruction [1].

\section{Conclusions}

To conclude, foreign body aspiration is not always a clear and easy diagnosis to make and may pose a diagnostic challenge to the clinician. It should be on the list of differential diagnoses for patients with unexplained respiratory symptoms or those with pneumonias that are recurrent or unresolving. Our patient describes a case of an elderly gentleman who was diagnosed initially with an unresolving pneumonia and later with possible malignancy; however, the foreign body was found incidentally on bronchoscopy, and the diagnosis was confirmed pathologically. The patient improved significantly after the foreign body was removed.

\section{Additional Information \\ Disclosures}

Human subjects: Consent was obtained by all participants in this study. Conflicts of interest: In compliance with the ICMJE uniform disclosure form, all authors declare the following: Payment/services info: All authors have declared that no financial support was received from any organization for the submitted work. Financial relationships: All authors have declared that they have no financial relationships at present or within the previous three years with any organizations that might have an interest in the submitted work. Other relationships: All authors have declared that there are no other relationships or activities that could appear to have influenced the submitted work.

\section{References}

1. Chen CH, Lai CL, Tsai TT, Lee YC, Perng RP: Foreign body aspiration into the lower airway in Chinese adults. Chest. 1997, 112:129-133. 10.1378/chest.112.1.129

2. Pritt B, Harmon M, Schwartz M, Cooper K: A tale of three aspirations: foreign bodies in the airway . J Clin Pathol. 2003, 56:791-794. 10.1136/jcp.56.10.791

3. Baharloo F, Veyckemans F, Francis C, Biettlot MP, Rodenstein DO: Tracheobronchial foreign bodies: presentation and management in children and adults. Chest. 1995, 115:1357-1362. 10.1378/chest.115.5.1357

4. Al-Majed SA, Ashour M, al-Mobeireek AF, al-Hajjaj MS, Alzeer AH, Al-Kattan K: Overlooked inhaled foreign bodies: late sequelae and the likelihood of recovery. Respir Med. 1997, 91:293-296. 10.1016/s09546111(97)90033-0

5. Steen K, Zimmermann T: Tracheobronchial aspiration of foreign bodies in children: a study of 94 cases . 
Laryngoscope. 1990, 100:525-530. 10.1288/00005537-199005000-00016

6. Limper AH, Prakash UB: Tracheobronchial foreign bodies in adults. Ann Intern Med. 1990, 112:604-609. 10.7326/0003-4819-112-8-604

7. Marik PE, Kaplan D: Aspiration pneumonia and dysphagia in the elderly . Chest. 2003, 124:328-336. 10.1378/chest.124.1.328

8. Surka AE, Chin R, Conforti J: Bronchoscopic myths \& legends: airway foreign bodies . Clin Pulm Med. 2006, 13:209-211. 10.1097/01.cpm.0000217598.29679.13

9. Sehgal IS, Dhooria S, Ram B, et al.: Foreign body inhalation in the adult population: experience of 25,998 bronchoscopies and systematic review of the literature. Respiratory Care. 2015, 60:1438-1448. 10.4187/respcare.03976

10. Hsu WC, Sheen TS, Lin CD, Tan CT, Yeh TH, Lee SY: Clinical experiences of removing foreign bodies in the airway and esophagus with a rigid endoscope: a series of 3217 cases from 1970 to 1996. Otolaryngol Head Neck Surg. 2000, 122:450-454. 10.1067/mhn.2000.98321

11. Zissin R, Shapiro-Feinberg M, Rozenman J, Apter S, Smorjik J, Hertz M: CT findings of the chest in adults with aspirated foreign bodies. Eur Radiol. 2001, 11:606-611. 10.1007/s003300000619

12. Rodrigues AJ, Oliveira EQ, Scordamaglio PR, Gregório MG, Jacomelli M, Figueiredo VR: Flexible bronchoscopy as the first-choice method of removing foreign bodies from the airways of adults. J Bras Pneumol. 2012, 38:315-320. 10.1590/s1806-37132012000300006

13. Blanco Ramos M, Botana-Rial M, García-Fontán E, Fernández-Villar A, Gallas Torreira M: Update in the extraction of airway foreign bodies in adults. J Thorac Dis. 2016, 8:3452-3456. 10.21037/jtd.2016.11.32

14. Lin L, Lv L, Wang Y, Zha X, Tang F, Liu X: The clinical features of foreign body aspiration into the lower airway in geriatric patients. Clin Interv Aging. 2014, 24:1613-1618. 10.2147/CIA.S70924

15. Ramos MB, Fernández-Villar A, Rivo JE, et al.: Extraction of airway foreign bodies in adults: experience from 1987-2008. Interact Cardiovasc Thorac Surg. 2009, 9:402-405. 10.1510/icvts.2009.207332

16. Alharthi BJ, Masoodi I, Almourgi MA, Alzahrani S: Occult foreign body in the lung mimicking bronchogenic carcinoma. Case Reports. 2014, 2014:2014207438. 10.1136/bcr-2014-207438

17. Mise K, Jurcev Savicevic A, Pavlov N, Jankovic S: Removal of tracheobronchial foreign bodies in adults using flexible bronchoscopy: experience 1995-2006. Surg Endosc. 2008, 23:1360-1364. 10.1007/s00464-008-01819

18. Dikensoy O, Usalan C, Filiz A: Foreign body aspiration: clinical utility of flexible bronchoscopy . Postgrad Med J. 2002, 78:399-403. 10.1136/pmj.78.921.399

19. Fang Y, Hsieh M, Chung F, et al.: Flexible bronchoscopy with multiple modalities for foreign body removal in adults. Plos One. 2015, 10:0118993. 10.1371/journal.pone.0118993 